\title{
Quality and Safety in Outpatient Laboratory Testing
}

\author{
Nancy C. Elder, MD, MSPH ${ }^{\mathrm{a}, *}$, \\ John Hickner, MD, MSc ${ }^{\mathrm{b}}$, Deborah Graham, MSPH ${ }^{\mathrm{c}}$
}

${ }^{a}$ Department of Family Medicine, University of Cincinnati, PO Box 670582, Cincinnati, OH 45267-0582, USA

${ }^{\mathrm{b}}$ Department of Family Medicine, The University of Chicago, Pritzker School of Medicine, 5841 S. Maryland Avenue, MC7110, Room M-160, Chicago, IL 60637, USA

${ }^{\mathrm{c}}$ National Research Network, American Academy of Family Physicians, 11400 Tomahawk Creek Parkway, Leawood, KS 66211-2672, USA

Clinicians in practice depend on laboratory testing to assist in diagnosing, treating, and monitoring their patients. Family physicians order laboratory tests on approximately $29 \%$ of the patients they see; internists order tests on $38 \%$ [1]. Although an increasing number of tests are performed in physicians' offices by their own staff, physicians still depend on outside laboratories for most of their testing needs. When it comes to the total testing process, most laboratory personnel have limited knowledge of the steps taken by physicians' offices, and similarly, most physicians and staff in physicians' offices have limited knowledge about the laboratory. We believe that improving testing quality begins with eliminating some of this ignorance.

Key points for improving quality are as follows:

- Most laboratory errors originate in the pre- and postanalytic phases

- The greatest opportunity for improvement in quality exists in pre- and postanalytic processes

- Critical areas include specimen collection, labeling, transport, data entry, and communication of results

- Gains in quality can be achieved by collaboration between clinicians and laboratorians

Most "laboratory errors" originate outside of the laboratory. They occur not in the analytic phase but in the pre- and postanalytic phases. Almost two

* Corresponding author.

E-mail address: eldernc@fammed.uc.edu (N.C. Elder). 
thirds of the errors occur in the preanalytic phase, including transport problems, which result in delayed or lost specimens, incorrect labeling, issues related to phlebotomy, and data entry errors [2,3]. Unfortunately, most studies that address preanalytic quality focus on hospital laboratories that care for hospitalized patients. Quality concerns regarding pre- and postanalytic steps as they relate to outpatient physician's offices have received little attention. This lack of attention represents a significant gap in quality data, because most medical care in the United States takes place not in the hospital but in the outpatient office setting. In a given month, for every eight patients who are hospitalized in the United States, 217 visit a doctor's office. More than one half of those visits are to primary care clinicians [4]. A recent Institute of Medicine report, "Patient Safety: Achieving a New Standard for Care," stressed the importance of focusing on outpatient care: "The consequences of medical errors in the (outpatient) setting - and the opportunities to improve-may dwarf those in hospitals" [5].

The goal of this article is to review the pre- and postanalytic testing process steps as they occur in primary care offices, with a special emphasis on the handoffs between physician's offices and laboratories. We review the literature and report on our own research on the testing process in primary care offices. By expanding the understanding of the testing process to include steps that occur outside the laboratory walls, we hope to encourage individuals involved in laboratory quality to consider all steps of the testing process in quality initiatives.

\section{The testing process in primary care}

The testing process begins and ends with the patient. We have developed a model of the testing process as it occurs in primary care offices, and we believe that the model also likely applies to specialty practices (Fig. 1). Clinicians assess their patients' complaints and conditions by obtaining a history, conducting a directed physical examination, and deciding whether laboratory or imaging tests are indicated. The test order is implemented in processes that often involve multiple staff in the clinicians' offices and the laboratories. While the test is being performed at the laboratory, results are tracked in the office until they are returned first to the office and then to the ordering clinician. The clinician reviews and responds to the results, documents this response in the medical record, and files the results for future use either electronically or in a paper chart. Patients are notified of the results and their meaning, appropriate clinical action is taken, and patients are monitored through any needed follow-up. This idealized testing process, however, often falls short in the realities of medical practice. Although a portion of primary care is provided within "closed" systems, in which primary care, specialty care, laboratory testing, and hospital care occur within integrated information and technology systems, most primary care is still provided by smaller, independent groups or individual providers. Most 


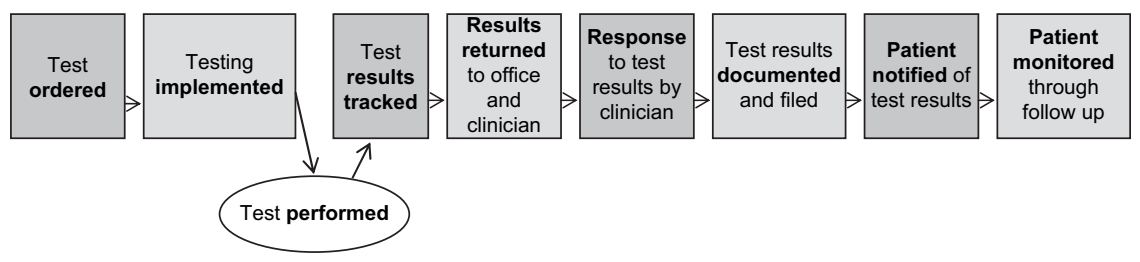

Fig. 1. The testing process in ambulatory office medicine. (Data from Hickner JM, Fernald DH, Harris DM, et al. Issues and initiatives in the testing process in primary care physician offices. Jt Comm J Qual Patient Saf 2005;31(2):81-9.)

primary care offices accept payment from multiple insurers. In 2001, 75\% of primary care offices reported having three or more managed care contracts, and one third of practices had more than ten [6]. Each insurer may require that a practice order test from a specific laboratory or hospital [7], which makes communication between the physician and laboratory more complex. Although technology could assist with this complexity, most primary care offices do not have electronic medical records; offices that do are not typically digitally connected to their reference laboratories [8-10].

\section{Errors and problems in the testing process in primary care offices}

For 8 months in 2004-2005, family physicians and their staff from eight practices anonymously reported errors related to the testing process to a research database of the American Academy of Family Physician's National Research Network (AAFP NRN). Participants submitted 590 error reports [11]. Eighteen focus groups were formed in these practices to discuss testing process problems and concerns [12]. The following discussion draws on this study to demonstrate the errors and problems that occur in all steps of the testing process in primary care offices.

\section{Ordering and implementing}

"It was a supervisor in the lab who told me wrong. It comes back to the day shift telling us one thing-and the afternoon doing another."

-Comment from a participant in the AAFP NRN study.

A study performed a decade ago in the Ambulatory Sentinel Practice Network noted that $56 \%$ of "laboratory errors" reported by family physicians occurred in the preanalytic phase [13]. Common sources of error included mislabeled specimens, improper specimen collection or handling, clerical problems, and ordering mistakes. Our recent research confirms that ordering and implementing steps account for $31 \%$ of testing process errors reported in family physicians' offices [11]. In a small number of technologically advanced offices, clinicians are able to enter laboratory orders directly into a digitally connected laboratory order system [14]. In most 
offices, however, the order is transmitted verbally or in written form several times before the order reaches the technician who performs the test. In hospitals, many tasks to implement a laboratory order are performed by laboratory personnel, including phlebotomy, specimen labeling, specimen preparation, and transportation. In office practice, however, these tasks are typically performed by office staff with varying backgrounds and training.

\section{Tracking and return of results}

"If the patient doesn't call and say, 'I haven't heard about my test results,' we really don't know that they're not back."

-Comment from a participant in the AAFP NRN study.

Many physicians' offices do not have adequate systems to monitor the flow of the laboratory tests they order. A 1996 survey of internists and family physicians found that only $17 \%$ of physicians reported having an effective test tracking system [15]. A 2000 study of Oklahoma family physicians noted that $78 \%$ of physicians had a tracking system but only $57 \%$ thought it was effective [16]. Difficulties with tracking include the complexity of how tests are ordered and implemented (they can be implemented at the time of the order, ordered now for implementation at a future time, or exist as a standing order), the number of laboratories used, the various ways in which test results are returned (digital, fax, mail, phone), and the competing demands on office practices that make test result tracking just one of many tasks performed to provide quality care to patients. Offices that do have test tracking systems find that these competing demands often lead to poor compliance with tracking protocols [12]. Physicians offices report that some test results never return from the laboratory. In a study of Colorado office practices, physicians reported that laboratory and radiographic reports make up half of the missing clinical information in their records [17].

\section{Clinician response to results and documentation}

"I think one of the biggest problems is the timeliness of the doctors...some are really bad, their charts just sit for weeks and weeks and weeks with the lab results, even though the nurses look at them first and know that they're pretty much normal, but normal isn't always normal."

-Comment from a participant in the AAFP NRN study.

In the AAFP NRN study, physicians and their staffs reported that $7 \%$ of all testing process errors related to incorrect, delayed, or inappropriate clinician responses [11]. Errors related to charting and documentation are also common and may account for as many as $15 \%$ of reported errors. These errors may relate to missing an important abnormal result or misinterpreting results in their clinical context or, more commonly (as noted in the previous quote), delay in responding to results. Results sometimes can 
be filed mistakenly in a patient's record with no review by a clinician. Again, laboratory test results are just one piece of clinical information with which clinicians and their staffs deal on a daily basis. Without organized systems and processes to manage these clinical data, important results may be overlooked.

\section{Patient notification and follow-up}

"In our practice, there are about 12 different systems for notifying patients.

Every doctor has their own way of doing that."

-Comment from a participant in the AAFP NRN study.

Clinicians and their staffs acknowledge that notifying patients of their results is inconsistent at best. In a 1996 survey of internists and family physicians, only $36 \%$ responded that they routinely notified all patients of abnormal results [15]. A more recent survey that documented the notification of abnormal pap smears and cholesterol levels found such documentation in patient charts only $47 \%$ and $67 \%$ of the time, respectively [18]. Although most patients want to be notified of their test results, even when normal [19], there is still a "no news is good news" attitude in some physicians' offices [12].

Following up with patients to ensure that the appropriate action has been taken based on the test result is even more difficult. Although a certain amount of responsibility for follow-up does rest with patients, clinicians have ethical and legal obligations to attempt to make sure patients get the follow-up care they need. Finding workable systems to ensure follow-up has been difficult $[11,12,15,16]$. As one participant in the AAFP NRN study put it, "Once the letter is mailed [to the patient with the results] it's kind of up to that patient."

\section{Handoffs between laboratory and physician's office}

Participants in our AAFP NRN focus group study gave us insights into why problems exist during the transitions between their office and laboratory. These problems occur with the ordering and implementation of the test and with the return of the test results. Three main contributing factors to these transitional problems were communication, technology interfaces, and conflicting policies and procedures.

\section{Communication}

That poor communication is a common factor in office-to-laboratory-tooffice problems is not surprising. A study of errors in family physicians' offices in Colorado found that communication problems were contributing factors in $71 \%$ of all reported medical errors [20]. Participants in our study cited problems with informal communication with laboratory personnel, 
such as when conflicting advice is received, and with formal communication. For example, a physician noted that "It amazes me how often you'll write an order and say 'Fax report to me as soon as possible' or you'll give them a pager number and say 'Page me' and that never gets done." Anyone who ever played the childhood game "telephone," in which a message is whispered person to person around a circle with the final message sounding nothing like the original one, can understand how the sheer number of people involved in these transitional testing process steps (eg, clinician, medical assistant, office clerical staff, laboratory clerical staff, medical technologist, laboratory manager) can lead to messages and information getting lost, delayed, or altered.

\section{Technology interfaces}

Although technology has the potential to greatly improve the transfer of data between the office and the laboratory [21], it also can be a source of error. Participant offices in our study included urban academic practices, suburban group practices, and rural community practices. None of our practices was in health systems that were fully digitally integrated with their laboratories or hospitals, and all had technology problems. As one physician noted, "You may or may not be surprised, it would literally take an act of God to get those computers to talk to each other... Even though it's all put in electronic format, and all potentially available instantaneously, it still comes in paper." When computer systems do interface with each other, it is not always successful. "We have a major problem with identifying who really ordered the test and that's really something beyond our control because we have to use a certain hospital lab which is really not designed to be an outpatient clinical lab but an inpatient hospital lab and they just have an archaic computer system and we tried really hard to change that and as of yet it really hasn't changed in 10 years."

\section{Conflicting or unknown policies and procedures}

When physician office staff are involved in implementing test orders, such as completing requisitions, drawing blood specimens, preparing specimens, and arranging for transportation, they may not always be aware of procedural changes at the laboratory. For example, a study participant describes an episode, "You'd order something the right way, you know that it's supposed to be a serum tube and the lab says 'Oh no, we need a lavender.' Well it didn't print up that you needed a lavender, and I've never had to give you a lavender for that before. You've got to call the patient back in and redraw it." Just as laboratory testing is only a small part of patient care for practices, the office-based client may be only a small part of the laboratory's workload, and up-to-date notification of procedural changes to the physician's office is unlikely to be a laboratory priority. 


\section{Ensuring safety and quality in the office-laboratory transitional steps}

Physicians' offices and laboratories have their work cut out for them to improve the quality of the testing process. Luckily, many individuals and organizations are working to find the best ways to do just that [21-24]. Some general patient safety principles, applied to the pre- and postanalytic transitional steps, might improve the safety and quality of the testing process, including the following steps:

- Standardization: This includes standardizing processes within a practice, within a laboratory and between offices and laboratories. Sometimes standardization can be community wide. For example, groups of physicians, insurers and laboratories may work together to standardize nomenclature for commonly performed tests and panels of tests, and develop requisition forms for each laboratory that use the same nomenclature and listing order. Physician's offices that use several laboratories could improve the quality of their test ordering when they don't have to navigate through many different forms and lists or deal with varying terminology. An office and a laboratory may standardize how test results are returned to the office, as well. If a laboratory both faxes and uses a computerized print out to the same office, the staff that collect and process the results will have more opportunities for error.

- Communication: From the office and laboratory sides, improved communication in the form of meetings, phone calls, and written and electronic memos can assist in preventing pre- and postanalytic problems. Putting human faces and names on contacts between laboratory and office can make asking questions, getting clarifications, and instituting new policies easier than when dealing with a generic "someone in the lab." Tracking concerns and problems from the laboratory and the office and arranging regular meetings to discuss common issues and solutions may prevent "unlabeled specimen," "incorrect tube," and "quantity not sufficient" calls to the office and "we never received the results" calls to the laboratory.

- Planned redundancy and back-ups: Although redundancy and efficiency hardly seem compatible terms, planned redundancies - the built in double checks and back-ups-usually save time by preventing errors and decreasing the amount of time spent scrambling to recover information. Planned redundancies include tracking systems for laboratory orders that use a log book and a copy of a requisition or a computerized lab $\log$ with a back-up computerized billing log, along with a system of oversight for reviewing log books. Laboratories could institute similar system of double checks for sending test results to physicians' offices.

- Safety culture: A safety culture has been defined by the Institute for Health Care Improvement as "an atmosphere of mutual trust in which all staff members can talk freely about safety problems and how to solve 
them, without fear of blame or punishment" [25]. Safety cultures are important in the laboratory and the physician office setting. People who work in health care, although occasionally responsible for making errors, also help prevent and ameliorate errors [26]. A safety culture seeks out individuals and the safety tasks they provide to incorporate such actions into the day-to-day functioning of medical practice.

\section{Summary}

Laboratory testing is an integral component of high-quality primary health care. For example, monitoring glycosolated hemoglobin levels in patients with diabetes, screening for lipid disorders in patients with heart disease, and performing Papanicolaou smears in adult women are just some examples of recommended primary care quality measures [23]. Primary care clinicians need a reliable laboratory to direct patient care. Threats to safety and quality pervade the testing process, however, with many of the risks occurring during the pre- and postanalytic phases during which specimens and information transfer between the laboratory and the physician's office. We believe that improvement begins with awareness that a problem exists and belief that the problem can be fixed. Laboratories and primary care physicians are working to improve quality in their respective fields, but greater gains can be achieved if quality efforts are integrated to include the entire testing process that begins and ends with the patient. We hope that this brief review of the testing process from the physician's office perspective can serve as an impetus for further innovations in testing quality.

\section{References}

[1] Woodwell DA, Cherry DK. National Ambulatory Medical Care Survey: 2002 summary. Adv Data 2004;346:1-44.

[2] Astion ML, Shojania KG, Hamill TR, et al. Classifying laboratory incident reports to identify problems that jeopardize patient safety. Am J Clin Pathol 2003;120(1):18-26.

[3] Plebani M, Carraro P. Mistakes in a stat laboratory: types and frequency. Clin Chem 1997; 43(8 Pt 1):1348-51.

[4] Green L, Fryer G, Yawn B, et al. The ecology of medical care revisited. N Engl J Med 2001; 344:2021-5.

[5] Aspden P, Corrigan J, Wolcott J, et al. Patient safety: achieving a new standard of care. Washington, DC: National Academies Press; 2004.

[6] Cherry D, Burt C, Woodwell D. National Ambulatory Medical Care Survey: 2001 summary. Advance data from vital and health statistics. Hyattsville (MD): National Center for Health Statistics; 2003.

[7] Sox HC. Saving office practice. Ann Intern Med 2003;139(3):227-8.

[8] Bates DW. Physicians and ambulatory electronic health records. Health Aff (Millwood) 2005;24(5):1180-9. 
[9] Boonstra A, Boddy D, Fischbacher M. The limited acceptance of an electronic prescription system by general practitioners: reasons and practical implications. New Technology, Work and Employment 2004;19(2):128-44.

[10] Loomis G, Ries J, Saywell R, et al. If electronic medical records are so great, why aren't family physicians using them? J Fam Pract 2002;51:636-41.

[11] Hickner J, Graham D, Elder N, et al. Testing process errors and their harms and consequences reported from family medicine practices: a study of the AAFP National Research Network. Qual Saf Health Care, in press.

[12] Elder N, Graham D, Brandt E, et al. The testing process in family medicine: problems, solutions and barriers as seen by physicians and their staffs. A study of the American Academy of Family Physicians' National Research Network. Journal of Patient Safety 2006;2(1): $25-32$.

[13] Nutting PA, Main DS, Fischer PM, et al. Toward optimal laboratory use: problems in laboratory testing in primary care. JAMA 1996;275(8):635-9.

[14] Simon SR, Kaushal R, Cleary PD, et al. Physicians and electronic health records. Arch Intern Med 2007;167:507-12.

[15] Boohaker EA, Ward RE, Uman JE, et al. Patient notification and follow-up of abnormal test results: a physician survey. Arch Intern Med 1996;156(3):327-31.

[16] Mold JW, Cacy DS, Dalbir DK. Management of laboratory test results in family practice: an OKPRN study. Oklahoma Physicans Resource/Research Network. J Fam Pract 2000;49(8): $709-15$.

[17] Smith PC, Araya-Guerra R, Bublitz C, et al. Missing clinical information during primary care visits. JAMA 2005;293(5):565-71.

[18] Poon EG, Gandhi TK, Sequist TD, et al. "I wish I had seen this test result earlier!" Dissatisfaction with test result management systems in primary care. Arch Intern Med 2004; 164(20):2223-8.

[19] Meza JP, Webster DS. Patient preferences for laboratory test results notification. Am J Manag Care 2000;6(12):1297-300.

[20] Fernald D, Pace WD, Harris DM, et al. Event reporting to a primary care patient safety reporting system: a report from the ASIPS Collaborative. Ann Fam Med 2004;2(4):327-32.

[21] Poon EG, Wang SJ, Gandhi TK, et al. Design and implementation of a comprehensive outpatient results manager. J Biomed Inform 2003;36(1-2):80-91.

[22] Hanna D, Griswold P, Leape LL, et al. Communicating critical test results: safe practice recommendations. Jt Comm J Qual Patient Saf 2005;31(2):68-80.

[23] Elder N. Quality and patient safety in the physician's office. FP essentials, edition no. 327, AAFP Home Study. Leawood (KS): American Academy of Family Physicians; 2006.

[24] Hickner JM, Fernald DH, Harris DM, et al. Issues and initiatives in the testing process in primary care physician offices. Jt Comm J Qual Patient Saf 2005;31(2):81-9.

[25] Institute for Healthcare Improvement. Patient safety: general. Available at: http://www.ihi. org/IHI/Topics/PatientSafety/SafetyGeneral. Accessed January 11, 2008.

[26] Parnes B, Fernald D, Quintela J, et al. Stopping the error cascade: a report on ameliorators from the ASIPS collaborative. Qual Saf Health Care 2007;16(1):12-6. 